\title{
Universal topological properties of shells in soap froth
}

\author{
K.Y. Szeto and W.Y. Tam \\ Department of Physics, \\ The Hong Kong Unsiversity of Science and Technology, \\ Clear Water Bay, Kowloon, Hong Kong
}

(Received 6 June 1995; revised manuscript received 3 October 1995)

\begin{abstract}
An analysis of the shell distribution function of two-dimensional soap froth reveals a universal topological relation on the average number $M(j, n)$ of sides per cell to the number of cells $K(j, n)$ in the $j$ th shell of a given center cell with $n$ sides. A plot of $M(j, n) K(j, n)$ vs $K(j, n)$ shows a slope of 5 for $j=1$ and a slope of 6 for $j \geq 2$, for all samples. The results are universal for soap froths in the scaling state with different preparations, different times, and different temperatures. A theoretical justification is given based on general topological arguments, which are independently supported by experiments.
\end{abstract}

PACS number(s): 82.70.Rr, 68.90.+g

Materials consisting of cellular structures such as metal grains and biological tissues are common in nature $[1,2]$. Among these systems, soap froth is considered as the paradigm for the study of trivalent two-dimensional cellular dynamics. Past experimental [3-8] and numerical [9-12] studies of the topological properties of soap froth are limited to the statistics of area (Lewis's law [13], von Neumann's law [14], and scaling law [6]) and nearestneighbor correlation (Aboav-Weaire law) $[15,16]$. So far, the evolution of soap froth after the scaling state can be explained by mean field theories $[17,18]$, indicating that correlation effects are not manifested in the analysis of area scaling law. However, more detailed analyses beyond the area scaling law have been done [19] and suggest strongly the importance of clarifying the role of correlation effects. Moreover, there has never been any experimental investigation on correlation effects beyond nearest neighbors in two-dimensional soap froth. Therefore, it is important both for the verification of the assumptions in mean field analysis and for the understanding of topological ordering processes in soap froth [20-25] to study correlation effects.

Soap froths are trivalent (three edges meeting at a vertex) two-dimensional cellular patterns. Phenomenlogically, soap froth admits the definition of shell distribution function. First, we define two cells as neighbors if they share a common edge. A cell $\beta$ belongs to the $j$ th shell of the center cell $\alpha$ if it is (i) a neighbor of an element of the $(j-1)$ th shell of $\alpha$ and (ii) not a neighbor of any element of the $k$ shell of $\alpha$ for all $k<j-1$. The first shell of the center cell $\alpha$ with $n$ sides consists of its $n$ neighbors. In Fig. 1 we show an experimental image of a two-dimensional soap froth shell structure around the center cell $\alpha$, labeled by 0 . The above definition admits defects, as indicated by the shaded cells in Fig. 1. The defect can be recognized as a cell that belongs to the $j$ th shell of $\alpha$, but that has either no neighbor belonging to the $k$ th shell of $\alpha$ for all $k>j$ or all its neighbors belonging to the $k(>j)$ th shell are themselves defects as in some pathological cases. These defects are cells that are blocked off from outer shells $[20,26]$.

We have analyzed data obtained from experiments with different conditions on soap froth. In one experiment the bubble chamber mounted horizontally was enclosed in a temperature bath whose temperature could be varied. The temperature of the cell was changed with different ramping rates. The experiment started with 2000 bubbles and was about 1000 bubbles when scaling was reached. Temperature ramping started only after the scaling state had been reached and the data we analyzed are for samples before breakage. For these samples, we find that the averaged area growth rate has a quadratic time dependence for all constant ramping rates used [27]. Moreover, scalings in side and area are still valid as observed in nonramping froths. In another experiment [28], the bubble chamber was left at room temperature and was mounted vertically for self-drainage of fluid due to the coarsening effect of the froth. Three

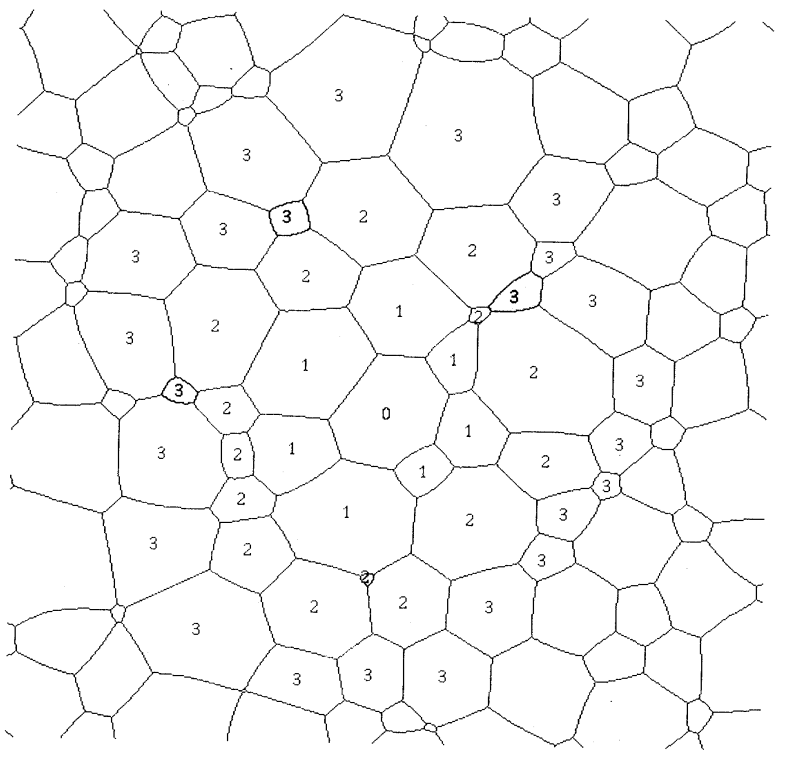

FIG. 1. Shell structure and defects in trivalent two-dimensional froth. The defects are shaded and the number denotes the shell from the center cell labeled 0 , which is a deformed heptagon. 
cell spacings were used to study the drainage effect. The experiment started with about 10000 bubbles and was about 5000 when scaling was reached. A high resolution digital charge coupled devise camera of $1037 \mathrm{X} 1344$ pixels was used to enhance spatial resolutions. The experiment shows a linear area growth rate after some drainage time and scalings are also observed. In the above experiments, 2-3 runs were carried out for each condition and good agreements were obtained.

From these experiments, we find a universal topological relation on the average number $M(j, n)$ of sides per cell to the number of cells $K(j, n)$ in the $j$ th shell of a given center cell with $n$ sides. For $j=1$, we get a linear plot of $K(1, n) M(1, n)$ vs $K(1, n)$ with a slope of $4.85 \pm 0.05$, which agrees with the Aboav-Weaire law. We generalize this investigation for $j>1$ and get a universal slope in
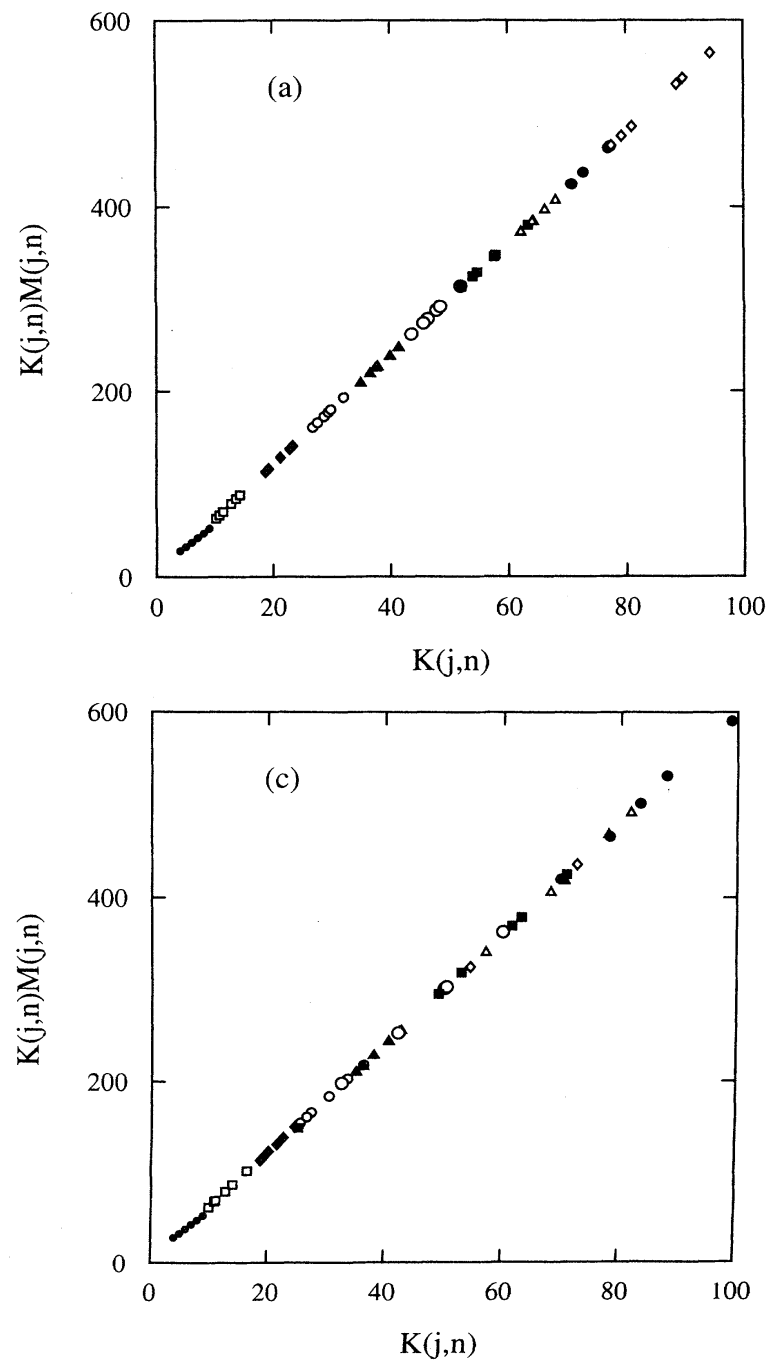

the $K(j, n) M(j, n)$ vs $K(j, n)$ plot for all $j \leq 10$ and all $n$, as shown in Fig. 2. The universal value of the slope is $6.00 \pm 0.01$ when all $j$ and $n$ values are used for the fitting. If we fit the slope for individual shell, i.e., fixing $j$, and obtain the slope for data for $4 \leq n \leq 9$, we get values of the slope as $6+\Delta(j)$, where $\Delta(j)$ is of the order of 0.1 , greater than zero for $j=2,3$, and oscillatory for larger $j$. The distinction of the slope for $j=1$ and $j>1$ is universal as the observation is made on many samples, including the same sample at different time in the scaling state, different samples with different gap thickness, and different samples heated at various rates. We also analyzed the average number $K(j, n)$ of cells belonging to the $j$ th shell of a center cell that has $n$ sides (Fig. 3). We find that $K(j, n)$ is approximately linear in $j$ for all samples
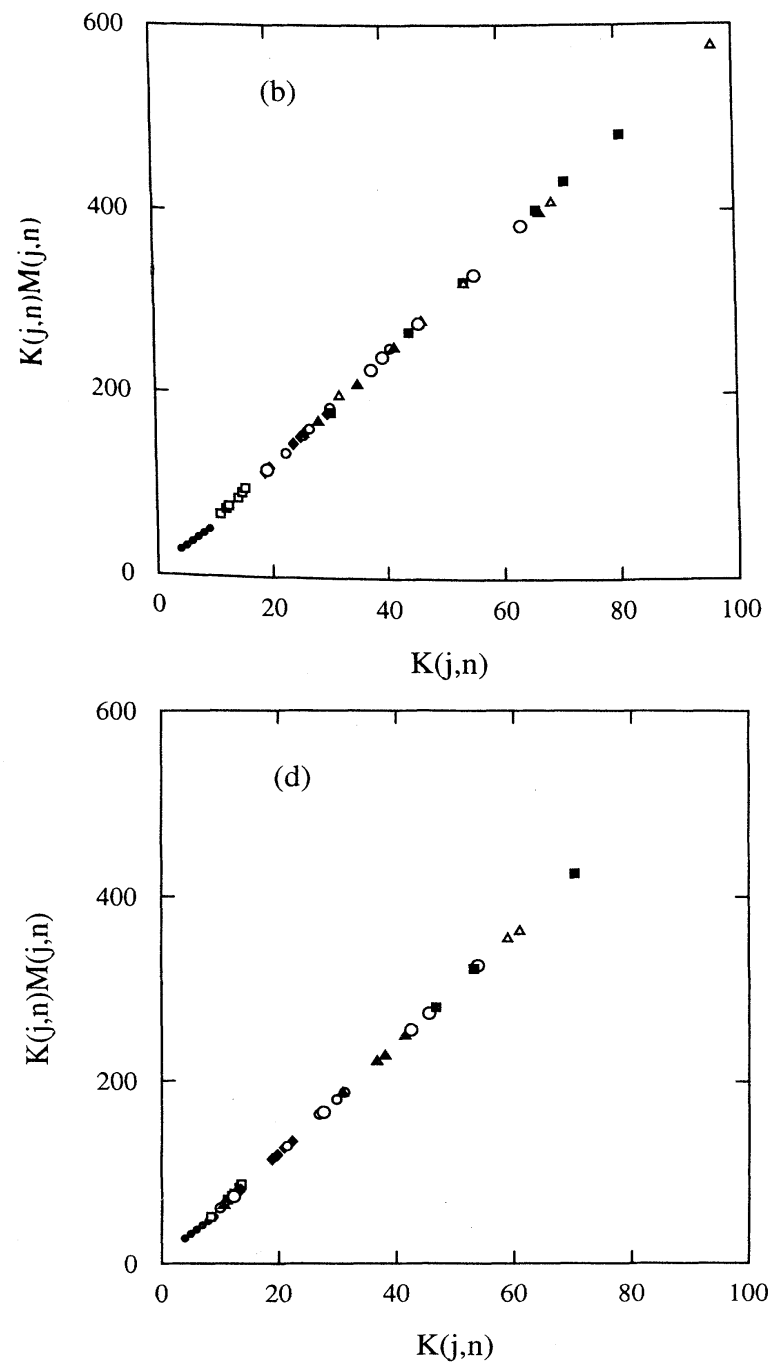

FIG. 2. Data collapse for the plot of $K(j, n) M(j, n)$ vs $K(j, n)$ at times (a) $2 \mathrm{~h}$ and (b) $14 \mathrm{~h}$ for constant temperature froths [28]. (c)-(f) are temperature ramping froths [27]: (c) is at time zero, temperature at $20^{\circ} \mathrm{C}$, and ramping rate $1{ }^{\circ} \mathrm{C} / \mathrm{h} ;(\mathrm{d})$ is $4 \mathrm{~h}$, temperature at $24^{\circ} \mathrm{C}$, and ramping rate $1^{\circ} \mathrm{C} / \mathrm{h}$. (e) is at time zero, temperature at $20^{\circ} \mathrm{C}$, ramping rate $4{ }^{\circ} \mathrm{C} / \mathrm{h} ;(\mathrm{f})$ is 1 $\mathrm{h}$, temperature at $24^{\circ} \mathrm{C}$, and ramping rate $4{ }^{\circ} \mathrm{C} / \mathrm{h}$. Symbols: $j=1$, full small circle; $j=2$, open square; $j=3$, full diamond; $j=4$, small open circle; $j=5$, full triangle; $j=6$, open circle; $j=7$, full square; $j=8$, open triangle; $j=9$, full circle; and $j=10$, open diamond. 

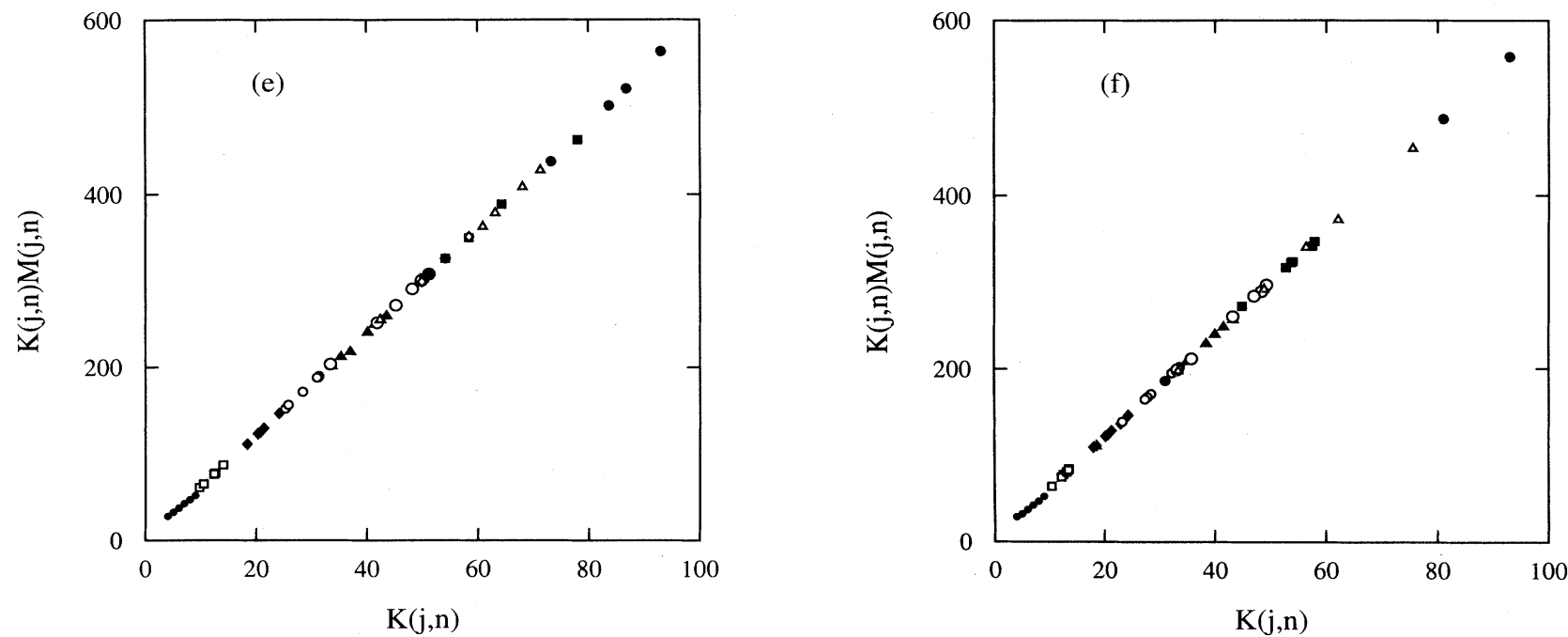

FIG. 2 (Continued).

$$
K(j, n)=A(n) j+B(n) .
$$

The intercept $B(n)$ depends linearly on $n$, but the slope $A(n)$ is insensitive to $n$. The linearity of $K(j, n)$ vs $j$ plot is understandable as $j$ is the topological distance and $K(j, n)$ is the circumference, which should be proportional to the radius $j$ in two-dimensional Euclidean space.

The universal feature that $M(j, n) \approx 6$ can be explained by the following relation. For $j \geq 2$,

$$
M(j, n)=4+\frac{K(j-1, n)+K(j+1, n)}{K(j, n)} .
$$

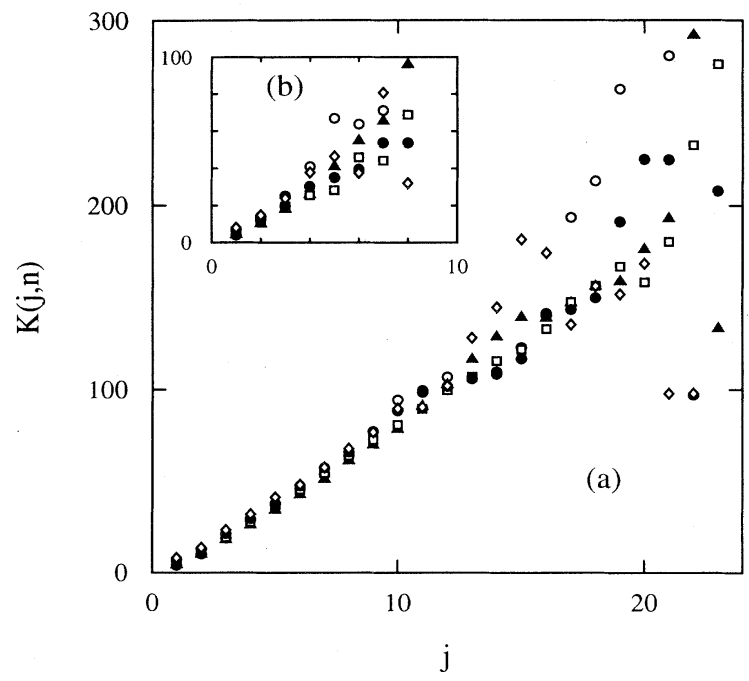

FIG. 3. Number $K(j, n)$ of cells in the $j$ th shell of a center cell of $n$ sides vs $j$. (a) $t=2 \mathrm{~h}$ and (b) $t=14 \mathrm{~h}$ for constant temperature froths. Symbols: $n=4$, open small circle; $n=5$, full triangle; $n=6$, open square; $n=7$, small full circle; $n=8$, open diamond.
Using the hypothesis of linear dependence of $K(j, n)$ on $j$ in this relation, we get immediately that $M(j, n)=6$ for $j \geq 2$.

We have a simple derivation for Eq. (2), assuming that there is no defect. Let $V^{+}(j, n)$ be the number of convex vertices in the $j$ th shell of the center cell $\mathrm{n}$, and $V^{-}(j, n)$ be the number of concave vertices, as shown in Fig. 4. The total number of vertices $N(j, n)=$ $V^{+}(j, n)+V^{-}(j, n)$ gives the total number of edges on the outer perimeter of the $j$ th shell. Note that the number of convex vertices in the $j$ th shell is the same as the number of cells in the $(j+1)$ th shell $V^{+}(j, n)=K(j+1, n)$. Now the total number of edges of all the cells in the $j$ th shell is $K(j, n) M(j, n)$. This quantity is also the sum of $N(j, n)$ for the outer perimeter, $N(j-1, n)$ for the inner perimeter, and $2 K(j, n)$ for the common edges shared by the cells in the $j$ th shell. Next, we also observe from

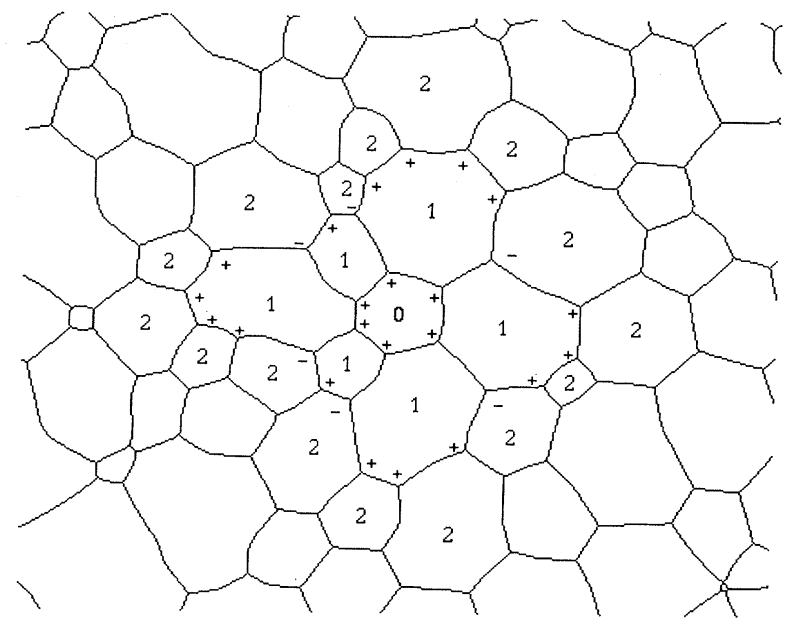

FIG. 4. Definition for $V^{+}(j, n)$ and $V^{-}(j, n)$. 
Fig. 4 that $V^{+}(j-1, n)=V^{-}(j, n)$, so that we have $K(j, n) M(j, n)=2 K(j, n)+N(j-1, n)+N(j, n)=$ $4 K(j, n)+K(j-1, n)+K(j+1, n)$, which is Eq. (2).

Experimentally, the linearity of $K(j, n)$ vs $j$ is tested in Fig. 3, which shows some scatters for large $j$, but is a good hypothesis for small $j$. The scatters in large $j$ are statistical and are due to the small number of center bubbles with large $j$. In fact, our samples have finite size and to select cells with large $j$ implies that we are looking only at the cells near the central region of the sample. As for Eq. (2), we have tested it independently and an error for $2[(\mathrm{RHS}-\mathrm{LHS})] /[(\mathrm{RHS}+\mathrm{LHS})]$ of less than $5 \%$ is observed (where RHS and LHS denote right- and lefthand side, respectively). For all samples that include different temperatures, times, thickness of the gap spacing between the plates that confine the two-dimensional froth, and different shells in a given sample, there is data collapse in the $K(j, n) M(j, n)$ vs $K(j, n)$ plot.

We will present analyses of data related to the topological charge and the idea of topological correlation length to separate dynamical correlation from geometric correlation in a different paper [19]. These analyses will shed some light on the observations of small and systematic deviations of $M(j, n)$, as manifested in $\Delta(j)$ in the fit for individual shell. The smallness of the deviations from 6 can be related to the presence of a small density of defects, which introduces small corrections to Eqs. (1) and (2). These deviations are systematic and are distinct from the voronoi construction of trivalent two-dimensional froth based on a random gas or perturbed hexagons. Nevertheless, the result $M(j, n) \approx 6.0$ for $j \geq 2$ in soap froth is universal, indicating that the assumption of negligible correlation effects beyond nearest neighbors is a good approximation. The importance of these experimental results will be seen in the shell model analysis of the topological properties of froths $[19,20]$, as well as in the theories of evolution of froths $[17,18]$ when more experimental features are to be explained.

We acknowledge discussions with T. Aste, G. LeCaer, E. Domany, H. Flyvberg, N. Rivier, and J. Stavans. W.Y.T. acknowledges support from the Direct Allocation Grant at HKUST.
[1] D. Weaire and N. River, Contemp. Phys. 25, 59 (1984).

[2] J. Stavans, Rep. Prog. Mod. Phys. 56, 733 (1993).

[3] J.A. Glazier and D. Weaire, J. Phys. Condens. Matter 4, 1867 (1992).

[4] J.A. Glazier and J. Stavans, Phys. Rev. A 40, 7398 (1989).

[5] J.A. Glazier, S.P. Gross, and J. Stavans, Phys. Rev. A 36, 306 (1987).

[6] J. Stavans and J.A. Glazier, Phys. Rev. Lett. 62, 1318 (1989).

[7] J. Stavans, Phys. Rev. A 42, 5049 (1990).

[8] J. Stavans, Physica A 194, 307 (1993).

[9] J.A. Glazier, M.P. Anderson, and G.S. Grest, Philos. Mag. B 62, 615 (1990).

[10] E.A. Holm, J.A. Glazier, D.J. Srolovitz, and G.S. Grest, Phys. Rev. A 43, 2662 (1991).

[11] T. Herdtle and H. Aref, J. Fluid Mech. 241, 233 (1992).

[12] J. Wejchert, D. Weaire, and J.P. Kermode, Philos. Mag. B 53, 15 (1986).

[13] D. Lewis, Anat. Rec. 38, 351 (1928).

[14] J. von Neumann, Metal Interfaces (American Society for Metals, Cleveland, 1952), p. 108.
[15] D.A. Aboav, Metallography 3, 383 (1970).

[16] D. Weaire, Metallography 7, 157 (1974).

[17] J. Stavans, E. Domany, and D. Mukamel, Europhys. Lett. 15, 479 (1991).

[18] H. Flyvberg, Phys. Rev. E 47, 4037 (1993); Physica A 194, 298 (1993).

[19] T. Aste, K.Y. Szeto, and W.Y. Tam (unpublished).

[20] M.A. Fortes and P. Pina, Philos. Mag. B 67, 263 (1993).

[21] R. Delannay and G. Le Caer, Phys. Rev. Lett. 73, 1553 (1994).

[22] G. Le Caer, J. Phys. A 24, 1307 (1991).

[23] M.A. Peshkin, K.J. Strandburg, and N. Rivier, Phys. Rev. Lett. 67, 1803 (1991).

[24] C. Godreche, I. Kostov, and I. Yekutieli, Phys. Rev. Lett. 69, 2674 (1992).

[25] F. Bolton and D. Weaire, Phys. Rev. Lett. 65, 3449 (1990); Philos. Mag. B 63, 795 (1991).

[26] T. Aste, D. Boose, and N. Rivier, Phys. Rev. E (to be published).

[27] W.Y. Tam and K.Y. Szeto, Phys. Rev. E 52, 877 (1996).

[28] W.Y. Tam and K.Y. Szeto (unpublished); K.Y. Szeto and W.Y. Tam, Physica A 221, 256 (1995). 


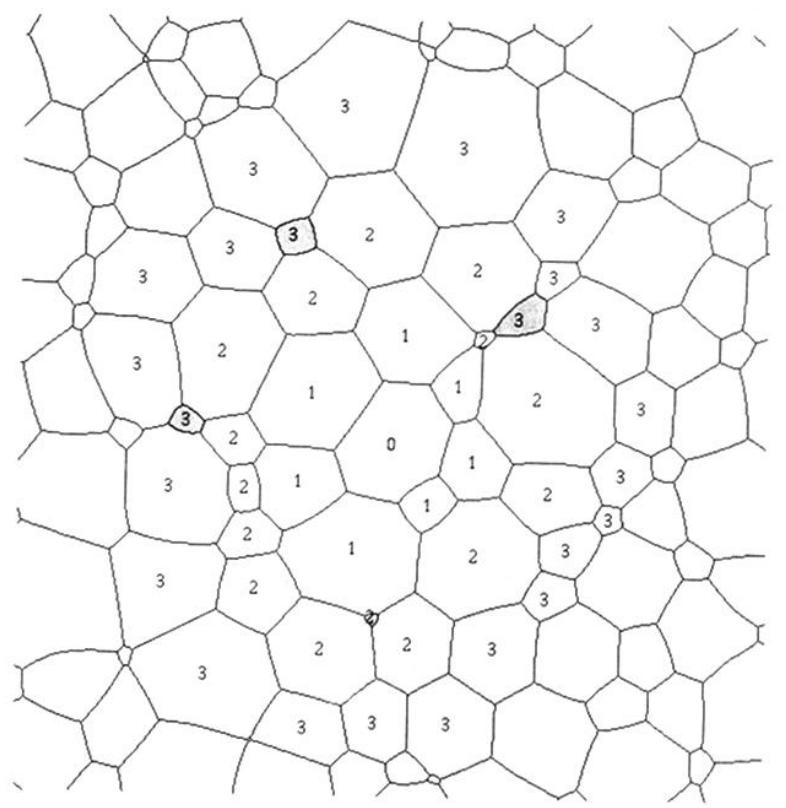

FIG. 1. Shell structure and defects in trivalent two-dimensional froth. The defects are shaded and the number denotes the shell from the center cell labeled 0 , which is a deformed heptagon. 